\title{
Fully automatic and precise data analysis developed for time- of-flight mass spectrometry
}

\author{
Stefan Meyer ${ }^{1}$, Andreas Riedo $^{1 \mathrm{a}}$, Maike B. Neuland ${ }^{1 \mathrm{~b}}$, Marek Tulej $^{1}$, Peter Wurz $^{1}$ \\ ${ }^{1}$ Space Research and Planetary Sciences, Physics Institute, University of Bern, Sidlerstrasse 5, CH - 3012 Bern, Switzerland \\ a currently: Sackler Laboratory for Astrophysics, Leiden Observatory, Leiden University, Niels Bohrweg 2, 2333 CA Leiden, The \\ Netherlands \\ ${ }^{b}$ currently: Swedish Institute of Space Physics, IRF, Rymdcampus1, SE-981 28 Kiruna, Sweden \\ E-mail: stefan.meyer@space.unibe.ch
}

\begin{abstract}
Scientific objectives of current and future space missions are focused on the investigation of the origin and evolution of the solar system with the particular emphasis on habitability and signatures of past and present life. For in situ measurements of the chemical composition of solid samples of the planetary surface, the neutral atmospheric gas, and the thermal plasma of planetary atmospheres, the application of spectrometers making use of time-of-flight mass analysers is a technique widely used. However, such investigations imply high statistics measurements and thus, a large amount of data to be analysed. Therefore, faster and especially robust automated data analysis with enhanced accuracy is required.
\end{abstract}

In this contribution, an automatic data analysis software, which allows fast and precise quantitative data analysis of time-of-flight mass spectrometric data is presented and discussed in detail. A crucial part of this software is a robust and fast peak finding algorithm with a consecutive numerical integration method allowing precise data analysis.

We tested our analysis software with data from different time-of-flight mass spectrometers and different measurement campaigns thereof. The quantitative analysis of isotopes, using automatic data analysis, yields results with a relative accuracy of isotope ratios up to $100 \mathrm{ppm}$ for a signal-to-noise ratio (SNR) of $10^{4}$. We show that the relative accuracy of isotope ratios is in fact proportional to $\mathrm{SNR}^{-1}$. Furthermore, we observe that relative accuracy of isotope ratio also is inversely proportional to mass resolution. Additionally, we show that the relative accuracy of isotope ratios is depending on the sample width $T_{\mathrm{s}}$ by $T_{\mathrm{s}}{ }^{-0.5}$. 


\section{Introduction}

Several future planetary missions are designed for exploration of the surfaces and atmospheres of different planetary objects, including ESA's ExoMars, [1] or JUICE, [2] and NASA's curiosity mission to mars, [3]. One of the key scientific objectives of these missions is the investigation of the formation of the solar system and its evolution, including the investigation of the processes, which are driving the exchange of material between planetary surface and exosphere, the alteration of both, thus all kind of space weathering processes. Measurements of the chemical composition of both, the gaseous exospheres and the surface material are a crucial piece of information to expand our knowledge about the origin of the solar system and to understand the processes in planetary evolution [4]. These data would also allow reconstruction of planetary climate, which may be of primary importance in the search for past or present life activities.

Laser ablation ionisation time-of-flight (TOF) mass spectrometry (LIMS) is regarded as one of the most promising analytical techniques for application in space research. Several miniature laser ablation ionisation mass analysers (with instrument name LAMS, LMS, LASMA) have been developed so far with the aim to use them for in-situ investigations of solid materials on planetary surfaces [5-8]. LIMS allows high measurement rates with high sensitivity and has a reasonably high mass resolution. It also allows probing at high lateral and vertical resolution [9]. The simplicity of operation, low power consumption and the robust design is of additional advantage for a space instrument. LIMS is a well-established analytical method for investigation of chemical composition of solid materials and applied in various research fields, including e.g. geology, metallurgy, environmental, and biological science [10]. Furthermore, TOF mass spectrometry is already used in several space missions. For instance ESA's Rosetta mission, where the RTOF instrument of the ROSINA experiment is built to analyse the chemical composition of cometary gas [11,12]. The Neutral Gas Mass Spectrometer (NGMS) was selected to detect and analyse the volatile species in the lunar soil on the Russian Luna-Resurs mission [13]. The Neutral gas and Ion Mass spectrometer (NIM), part of the Particle Environment Package (PEP) on-board ESA's upcoming L-class mission JUICE, is intended for composition measurements of neutral gas and thermal plasma of Jupiter's moons [14]. With these instruments, i.e., the LMS, NGMS and NIM, new capabilities were opened to study planetary exospheres and solid sample from the surface. By analysing rocks on a surface as radio-isotope geochronology with precise measurements of e.g. $\mathrm{Pb}$ isotopes is feasible, or the investigation of mineralogy of rocky planetary materials due to very sensitive elemental analysis. However, such investigations imply high amounts of measurements, often with different measurement conditions. For example, for establishing mineralogical maps of the surface of a solid sample requires measurements in the defined region of interest on the sample with a sufficiently high spatial resolution. Depending on sampling rate and measurement time available and the resolution required, these can be about $\sim 10^{3}$ measurements in a $\mu \mathrm{m}$ to $\mathrm{mm}$ sized region $[15,16]$.

For such large data sets manual data reduction and processing is not feasible. While being very time consuming, it is furthermore important that reproducibility and robustness of the results is provided, also for different analysts working on the same project. Therefore, faster and especially automated data analysis with high accuracy is required. Based on these needs, we developed a robust automatic data analysis software, which allows the guided data analysis of TOF data. The analysis procedure follows the sequence of data read-in and accumulation procedure up to the quantitative results of the abundances of elements and isotopes within minutes. Thereby, we present a robust and fast numerical integration method, which allows all in all a precise and fast data analysis. We tested our data analysis software in several different measurement campaigns with the LMS instrument [15-22]. In addition, it was also used for data analysis with the NGMS instrument prototype [13]. Currently, this automatic data analysis software is used in development and testing of the NIM instrument [14]. 


\section{Experimental methods}

\subsection{NIM prototype instrument}

The results presented in Sec. 4 are obtained with the NIM prototype instrument. NIM is a TOF instrument with heritage from the RTOF instrument of the ROSINA experiment on the Rosetta mission [11,12] and the PBACE (Polar Balloon Atmospheric Composition Experiment) instrument [23]. NIM is designed to operate in three different modes: thermal mode, neutral mode and ion mode. In neutral mode, the ambient gas enters the ionisation region of the ion source directly (open source), in thermal mode the ambient gas enters through an ante-chamber (equilibrium sphere) where the gas is thermally accommodated with the chamber wall before it proceeds to the ionisation region of the ion source (closed source). In ion mode, the ambient thermal ions are measured directly. A detailed description of the NIM prototype instrument and the results from an extensive test and measurement campaign are given in [14].

For the herein presented results, measurements conducted in thermal mode are used. In thermal mode, the neutral gas is decelerated from beam velocity down to thermal energies in an equilibrium sphere, and passed on into the ion source in thermal state, where it is ionised by an electron beam and stored [24], until the subsequent guidance through the ion optics to the multi-channel plate (MCP) detector. This mode (also referred to as closed source in the literature) is used for neutral gas measurements at any JUICE mission phase, but mainly during Europa torus crossing and all other flybys. The so measured neutral gas beam consisted of a gas mixture of $89.5 \% \mathrm{H}_{2}, 7.5 \% \mathrm{Ne}, 1.5 \% \mathrm{Ar}$ and $1.5 \% \mathrm{Kr}$ with a beam velocity of $2.4 \mathrm{~km} \mathrm{~s}^{-1}$.

\subsection{Data Acquisition}

The analogue signal from the MCP detector of the NIM prototype instrument is acquired and digitised by standard high speed analogue-to-digital converter (ADC) data acquisition card U1084A from Keysight, former Agilent. This card can digitise with up to $4 \mathrm{GS} / \mathrm{s}$ with an analogue bandwidth of up to $1.5 \mathrm{GHz}$ and a vertical resolution of 8 bit for a single waveform. The averager firmware of the data acquisition card allows the on-board accumulation of single waveforms in real time. In standard acquisition mode, the waveforms from about 1000 sequences are accumulated online and stored as one measurement file. Typically, 100 measurement files, summed up to one spectrum, were recorded with $20 \mu \mathrm{s}$ TOF, which corresponds to the mass range from 0 to approximately $800 \mathrm{u} / \mathrm{e}$.

\section{Data Reduction and Mass Spectrometric Analysis}

\subsection{Data Acquisition Process}

The mass spectrometric analysis of a TOF spectrum involves waveform accumulation, spectrum calibration and appropriate representation of the spectral intensities for determination of the quantitative information. Accumulation of waveforms is performed to statistically enhance the dynamic range and the spectrum calibration to obtain the mass scale from the TOF spectrum. For acceleration of the mass calibration and for making this analysis step independent from the individual experimenters, we developed an algorithm for automatic mass calibration. This algorithm is based on the identification of mass peaks occurring at approximately multiples of the integer atomic mass unit and the identification of isotope patterns of incisive elements. Finally, all these operations allow the quantitative analysis of isotopes by comparing the number of measured ions of different isotopes within one particular element, or the quantitative elemental analysis by comparing the number of measured ions for the isotopes of different elements. 
In spite of the very explicit data analysis steps to be applied, the following points are important features of any measured spectrum.

\section{Background}

In general, a certain slowly varying background, mainly caused by DC voltage drifts on the anodes, can be observed in every measurement. This leads to a shifting baseline where mass peaks can occur in an ascending region, a straight region or a descending region respectively. Therefore, background subtraction has to be done before peak area determination. An example of such a background is illustrated in Fig. 1 by its grey shaded area in a measurement of different noble gases with the NIM prototype. The shape of the background can be different for diverse instrument settings, including e.g. voltages of the ionoptical system and ionisation parameters.

\section{Peak Asymmetry}

Theoretically, Gaussian-shaped mass peaks of ions produced by electron impaction ionisation from a thermal gas, with a normal kinetic energy distribution from the ion source can be expected. However, in practice also asymmetric peaks can occur. This asymmetry might be caused during the ionization process itself, or by the ion-optical system that is not fully optimized. In addition, at high ion densities the mass peaks could be broadened due to mutual coulombic repulsion within an ion packet [17]. Also, RC low pass time constants in the detector circuit and acquisition electronics can lead to an exponentially decreasing function.

\section{Noise}

Basically, in every electronic measurement system a certain noise amplitude has to be considered. Fig. 1 shows an example of the noise level in a measurement with the NIM prototype of different noble gases, with an inset on two subsequent mass peaks.

Generally, one measurement contains several waveforms to enhance dynamic range by statistical means, which are automatically generated and saved by the data acquisition system. These consecutively acquired files with the effective measurement data as a digital value for each sampling point are then read in by the data analysis software.

Thereof, the intensity for one waveform, which is the back conversion of the digital values to the corresponding measured voltage $U(T O F)$, can be calculated as the detected (counted) electron rate $N_{e}$, which is the number of electrons per time increment with the unit [electrons/s], using

$$
N_{e}(T O F)=\frac{U(T O F)}{R \cdot e}
$$

with $R$ the input impedance $\left(R=50 \Omega\right.$ in our case) of the acquisition circuit and $e=1.602 \cdot 10^{-}$ ${ }^{19}$ As the electron charge.

To link the peaks in the TOF spectrum to its corresponding masses, conversion from TOF to mass scale has to be performed. In TOF mass spectrometry, separation between ions of different masses is achieved along the field free drift path, after accelerating them to a certain kinetic energy. The mass-per-charge ratio $\mathrm{m} / \mathrm{q}$ can be written as

$$
\frac{m}{q}=k_{0} \cdot\left(t-t_{0}\right)^{2}
$$

with the spectrometer constants $k_{0}$ and $t_{0}$, which are instrument dependent [25]. $k_{0}$ and $t_{0}$ can be determined by a linear fit of the TOF for identified isotopes against the square root of their respective masses [18]. With a suitable set of isotopes, mass scale accuracies better than 500 ppm can be achieved [26]. 


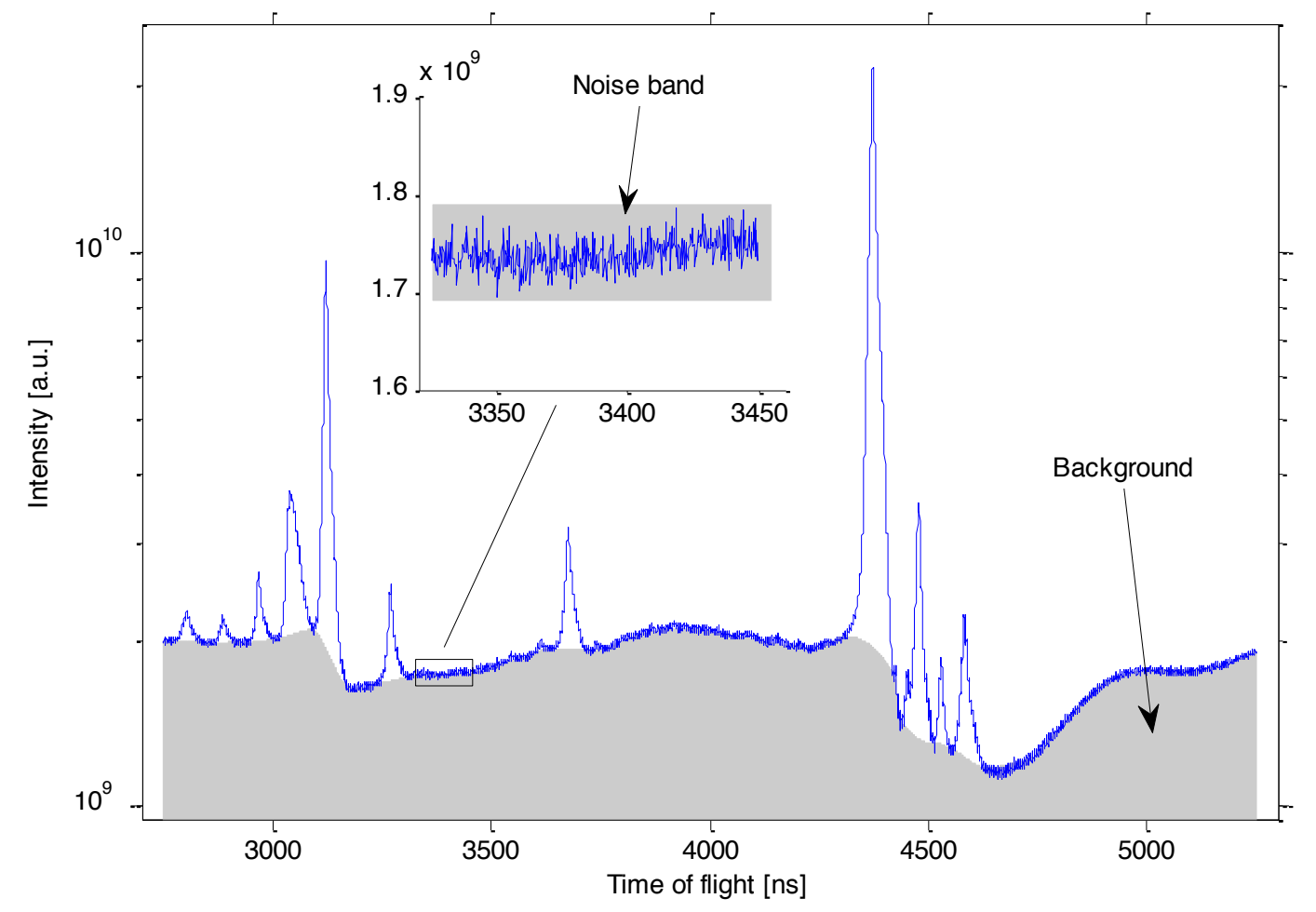

Figure 1: Example of background in a measurement of different noble gases with the NIM prototype. The inset shows the noise band of this measurement with a zoom on the corresponding baseline.

\subsection{Peak Area Determination}

Quantitative element and isotope analysis generally deals with abundances of particular species. Therefore, one is interested in the number of ions of such species, i.e., elements and/or isotopes. However, using Eq. 1, the number of electrons of a particular isotope $A_{e}^{[a, b]}$, with its mass peak distributed in the TOF interval $[a, b]$ can be calculated by its area as

$$
A_{e}^{[a, b]}=\int_{a}^{b} N_{e}(T O F) d T O F \propto A_{i o n}^{[a, b]},
$$

which is then proportional to the number of ions of this particular isotope $A_{\text {ion }}{ }^{[a, b]}$, assuming constant gain for the MCP-detector. This is well the case, since the MCP voltage is kept at a specific potential during measurements.

Peak area determination according Eq. 3 can be done by different approaches such as numerical (direct) integration and peak fitting either peak by peak or multiple peaks at the same time. With respect to a robust automatic algorithm, one should keep peak area determination as simple as possible and do single peak analysis by calculating the peak areas one after another. As has been shown in [19], multiple peak fitting lacks of robustness, due to the growing number of parameters to be estimated. Furthermore, intermediate and interfering isotopes, which have to be assigned correctly, makes fitting of multiple peaks even more problematic for an automated analysis.

\section{Peak Window Finding}

Nevertheless, also for single peak analysis one has to find the appropriate peak window, i.e., the TOF-interval $[a, b]$ where the corresponding mass peak is located in. Therefore, possible mass peaks have to be identified within the mass spectrum and its starting point $a$ and ending point $b$ have to be determined very accurately. Currently, most algorithms used in mass spectrometry are based on local maxima searching methods for peak detection. On the other hand, several algorithms using wavelet transform methods were proposed [27]. The continuous wavelet transform-based pattern matching algorithm presented in [27] seems to 
be very powerful, in this case for mass spectra obtained by surface enhanced laser desorption ionisation time-of-flight (SELDI-TOF) spectroscopy. Yet, it has to be mentioned that these mass spectra consisted of a dynamic range of about one to two decades and peaks were identified for $S N R>3$. The requirement for data analysis to detect and accurately quantify major elements as well as trace elements (down to lowest SNR) within a dynamic range of about $10^{5}$ makes the situation more complex. Furthermore, wavelet transform methods are only used for peak identification, the interval boundaries $a$ and $b$ are not given by this process.
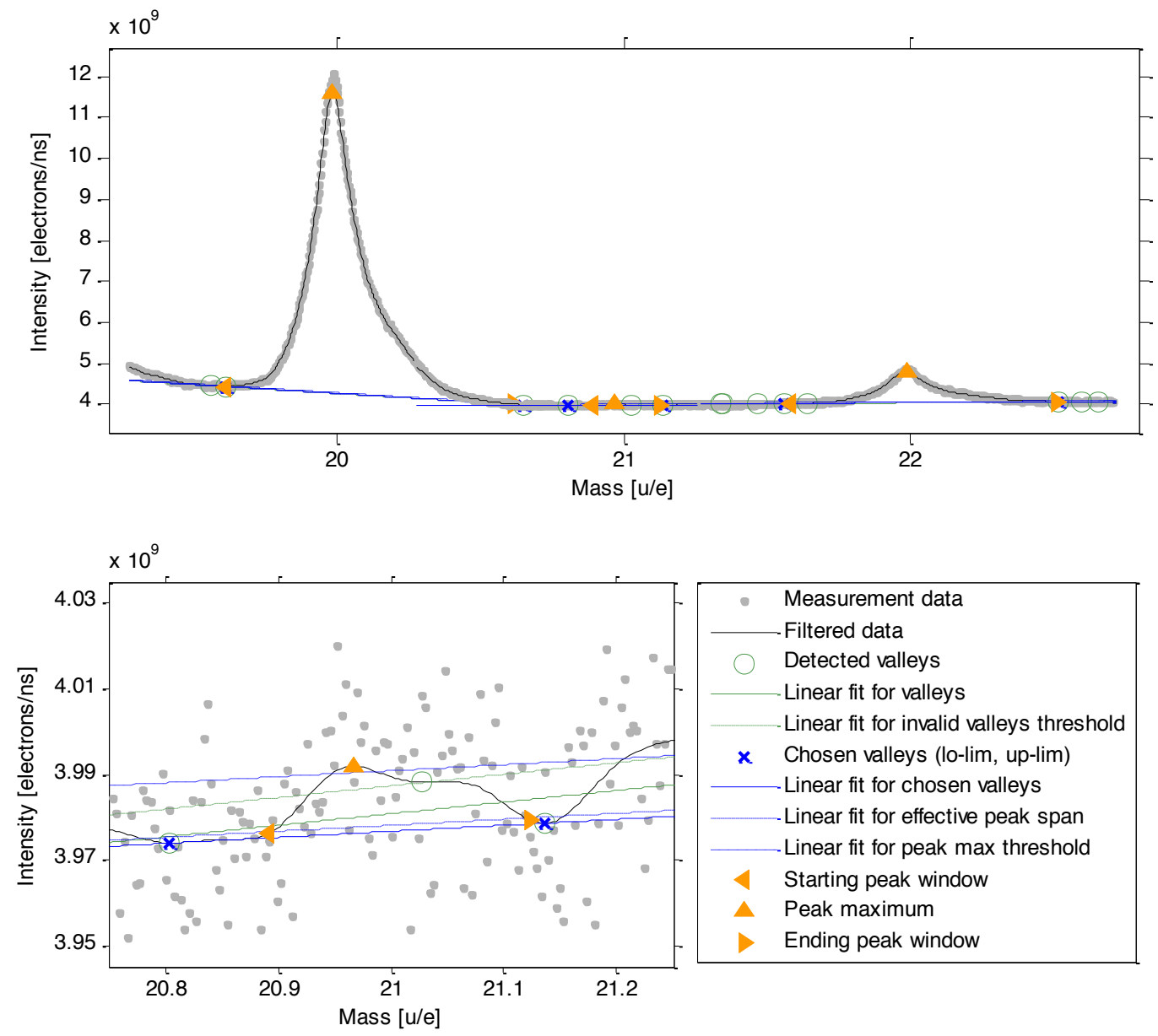

Figure 2: Top panel shows the peak window finding for the ${ }^{21} \mathrm{Ne}$ isotope peak near the noise limit. In bottom panel, the peak window finding for the isotopes ${ }^{20} \mathrm{Ne},{ }^{21} \mathrm{Ne}$ and ${ }^{22} \mathrm{Ne}$ is given.

For determining the correct peak windows, the raw measurement data are pre-processed with an adaptive moving average filter. In our analysis software, we apply a $1^{\text {st }}$-derivative zero-crossing algorithm to the filtered data for finding the peaks and the valleys between the peaks. The peak areas are calculated by using the raw, thus non-filtered data to avoid possible influences of the pre-processing of the data.

The implemented process of finding the peak windows is illustrated in Fig. 2. The lower panel of Fig. 2 shows the mass range of $\mathrm{Ne}$ with the detected $\mathrm{Ne}$-isotope peaks and the boundaries of the peaks. The definition of peak boundaries is most complicated for peaks that are close to the noise limit. As an example for an extreme case, the detail of Fig. 2 shows the mass region of the ${ }^{21} \mathrm{Ne}$ isotope with the details of the different steps of finding the peak boundaries of this low intensity mass peak near the noise limit. First, the local maximum located nearest to the theoretical mass of the according mass peak is selected as the centre of the peak. Then, a linear fit of the detected valleys within an expected mass range around this peak centre is executed (Fig. 2, linear fit for valleys). The two valleys next 
to the peak centre, which are below the linear fit added with $0.35 \sigma$ error of fit and $0.3 \sigma$ of noise are selected as potential peak window boundaries (linear fit for invalid valleys threshold in Fig. 2). This linear fit and the threshold defined by the fit avoid the false allocation of valleys in case of possible notches in the peak. In the case of ${ }^{21} \mathrm{Ne}$, shown in Fig. 2, it can be seen that a detected valley was decided to be a false one, due to the procedure described above. The potential peak window boundaries are linearly fitted again (linear fit for chosen valleys in Fig. 2), i.e., its linear interpolation with additional $0.1 \sigma$ of noise is used to reduce the peak window boundaries to the nearest sample point next to the interceptions with the filtered data (linear fit for effective peak span in Fig. 2), thus giving exact starting point $a$ and ending point $b$ of the peak. In addition, this linear interpolation with additional $1 \sigma$ of noise serves as threshold for peak detection (linear fit for peak max threshold in Fig. 2).

\section{Background Correction}

Due to the varying background, the slopes of the linear fits for effective peak span are not the same (see Fig. 1, Fig. 2) for all isotopes, respectively all elements in the mass spectrum. This needs to be considered by using a suitable background model for precise peak area calculation.

We tested different background correction models in the past (constant, linear, quadratic and exponential). It turned out that best quantitative results are obtained with a linear correction model for the local background of each mass peak. Furthermore, the background area error is about two orders of magnitude lower than the peak area calculation error. Therefore, a linear background correction is implemented peak-wise from starting point $a$ to the ending point $b$ of the determined peak window.

\section{Signal-To-Noise Ratio and Mass Resolution}

The signal-to-noise ratio SNR of a mass peak is calculated as

$$
S N R=\frac{I_{p}^{\max }}{\operatorname{std}\left(I_{b}\right)}
$$

where $I_{p}^{\max }$ denotes the background corrected peak's maximum intensity and $\operatorname{std}\left(I_{b}\right)$ describing the standard deviation of the baseline intensity over an equivalent mass range as the peak itself, measured at the end of the spectrum where no mass peaks are present (as representative of the noise level within the spectrum). Equation 4 is in accordance with a technical note from Agilent Technologies about signal, noise and detection limits in mass spectrometry [28].

Mass resolution $\mathrm{m} / \Delta \mathrm{m}$ is the ability to distinguish between two mass peaks (i.e., between ions with adjacent mass-to-charge ratio) and can be written as

$$
\frac{m}{\Delta m}=\frac{1}{2} \frac{t}{\Delta t}=\frac{1}{2} \frac{\mu_{t}}{F W H M_{t}}
$$

where $t$ denotes the TOF with $\mu_{t}$ the centre of the intensity peak and $\Delta t$ is determined experimentally from the TOF spectrum taking the width of the intensity peak at full width at half maximum (FWHM), [10]. This peak width includes the uncertainty of the time of ion formation in the source (duration of ion formation in the source, ion location in the source, various initial conditions of the ions), broadening effects caused by ion optics during the ion flight (Coulomb repulsion), broadening effects in the reflectron (e.g. field imperfections) and duration of the signal generation by the MCP-detector. The resolving power of the mass spectrometer is in principle a function of all these effects and choice of the experimental conditions can be critical for achieving an optimal mass resolution.

With the choice of the peak-wise area determination and especially its peak window finding, a minimum mass resolution is necessary such that the mass peaks are completely separated. Otherwise, the peak window and thus the area of interfering mass peaks would be underestimated by falsely cutting off too much of background. Hence, the minimum mass resolution $(\mathrm{m} / \Delta \mathrm{m})_{\min }$ is defined with a maximum span of $1 \mathrm{u}$ to sufficiently separate two peaks 
separated by $1 \mathrm{u}$ without interference, corresponding to approximately $\pm 3 \sigma_{\max }$ of an adequate Gaussian peak. This means $1 \mathrm{u}$ corresponds totally to $6 \sigma_{\max }$ and leads to

$$
\left(\frac{m}{\Delta m}\right)_{\min }=\frac{\mu_{m}}{F W H M_{\max }}=\frac{\mu_{m}}{2 \sqrt{2 \cdot \ln (2) \cdot} \cdot \sigma_{\max }}=\frac{6 \cdot \mu_{m}}{2 \sqrt{2 \cdot \ln (2)}}
$$

with $\mu_{m}$ as centre of the mass peak. This means the minimum mass resolution linearly increases with the peak's mass. This has to be kept in mind especially for the interpretation of the calculated accuracies of the isotope ratios and will be discussed in detail in Sec. 4.3.

\section{Peak Area Calculation}

For mass spectrometric analyses, the area of each individual mass peak needs to be derived. The question remains, if peak area calculation by applying a fit or by numerical integration is to be preferred. Already in the past, we tested our analysis software with different numerical integration methods and fitting functions, such as Gaussian fit, EMG fit, numerical integration using spline interpolation and numerical Monte Carlo (MC) acceptance/rejection integration to compare the performances [19]. Therein, we presented our results on peak areas derived by different integration methods for measurements on standard materials. We showed that higher accuracy results are obtained with numerical integration methods compared to least-squares fitting methods. These observations are in agreement with theoretical considerations discussed in [29], where the authors state that the least-squares method consistently underestimates the area of a fitted curve in case of Poisson statistics. Moreover, by assuming the peak shape matching a predefined mathematical function, a preselection is made that is not necessarily in accordance with the measured peak shape. The disadvantage of numerical integration using spline interpolation is that it does not give any error estimation. Testing peak integration with MC integration was proven to not being suitable for our purposes, due to the much longer calculation times in comparison to the other methods mentioned before [19].

For a robust and fast automatic data analysis, we propose a different type of numerical integration method. The consecutively called Simpson integration is based on Simpson's classical 3/8-rule for the integration of equally spaced abscissas [30]. The mathematical details are discussed in [30]. The corresponding formula for area calculation is

$$
\begin{gathered}
A_{i-(i+3)}=\int_{x_{i}}^{x_{i+3}} f(x) d x=T_{s} \cdot\left[\frac{3}{8} f\left(x_{i}\right)+\frac{9}{8} f\left(x_{i+1}\right)+\frac{9}{8} f\left(x_{i+2}\right)+\frac{3}{8} f\left(x_{i+3}\right)\right]+\mathcal{O}\left(T_{s}{ }^{5} f^{(4)}\right), \\
x_{i}=x_{0}+i \cdot T_{s}, \quad i=0,1, \ldots, N
\end{gathered}
$$

where $T_{s}$ represents the constant sample width, $x_{0}$ the first sample, $x_{i}$ the $i^{\text {th }}$ sample, $f\left(x_{i}\right)$ the intensity of the data to be integrated at position $i, f^{(4)}$ the $4^{\text {th }}$-derivative of the intensity function and $A_{0-3}$ denotes the area of the first step, using samples $x_{0}$ to $x_{3}$. This step can easily be extended over the interval $x_{i}=[a, b]$, where $a$ and $b$ describe the TOF of peak start and end, by stepwise summing up $A_{i-(i+3)}$ to obtain the area of the peak $A_{[a, b]}$. The error of this numerical integration, denoted as $O\left(T_{s}^{5} f^{(4)}\right)$ in Eq. 7 depends on the sample width $T_{s}^{5}$ and the $4^{\text {th }}$ derivative of the intensity function $f(x)$ and can be written as

$$
\epsilon=\left|\frac{3}{80} T_{s}^{5} f^{(4)}(\xi)\right|, \quad \xi \in[a, b] .
$$

With Eq. 8 a maximal error $\varepsilon$ for the numerical integration can be estimated by taking the maximum of the $4^{\text {th }}$-derivative of the measured intensity in interval $[a, b]$ as an upper limit,

$$
\epsilon \leq \frac{3}{80} T_{s}^{5} \cdot \max _{a \leq x \leq b}\left|f^{(4)}(x)\right| .
$$

Computing the $4^{\text {th }}$-derivative numerically, using finite differences [31], the maximum numerical error estimate $\varepsilon_{\text {Simpson }}$ is

$$
\epsilon_{\text {Simpson }} \leq \frac{3}{80} T_{S}^{5} \cdot \max _{a \leq x_{i} \leq b}\left|\frac{f\left(x_{i}\right)-4 f\left(x_{i+1}\right)+6 f\left(x_{i+2}\right)-4 f\left(x_{i+3}\right)+f\left(x_{i+4}\right)}{T_{s}^{4}}\right| .
$$


As described above, with the time integral over the electron rate in Eq. 3, the peak area is nothing else than the counted and accumulated number of electrons over a certain TOF interval $[a, b]$ (corresponding to the number of detected ions). This process is subject to the Poisson distribution. Therefore, the following area and error considerations are valid, in accordance with [29]: Assuming three independent functions, $f_{p}(t)$ describing the effective peak signal (from ions only), $f_{b g}(t)$ a background signal and a function $f_{n}(t)$ for the remaining noise, composing the total signal to be measured $f_{\text {tot }}(t)$,

$$
f_{\text {tot }}(t)=f_{p}(t)+f_{b g}(t)+f_{n}(t)
$$

Then, the area $A_{p}$ within peak interval $[a, b]$ is given by

$A_{p}=\int_{a}^{b} f_{\text {tot }}(t)-f_{b g}(t)-f_{n}(t) d t=\underbrace{\int_{a}^{b} f_{\text {tot }}(t) d t}_{A_{t o t}}-\underbrace{\int_{a}^{b} f_{b g}(t) d t}_{A_{b g}}-\underbrace{\int_{a}^{b} f_{n}(t) d t}_{A_{n} \approx 0}=A_{\text {tot }}-A_{b g}$,

where the integral over the noise function $f_{n}(t)$ should evaluate to zero, assuming random distributed noise. Using Poisson statistics, where the variance of a stochastic variable is equal to its expectation value [32], and Gaussian error propagation, the variance of the peak area $\sigma_{A p}^{2}$ can be derived with the statistical variance of the total area $\sigma^{2}$ Atot and $\sigma^{2}{ }_{A b g}$ the variance of the background area. This results in an estimate $\sigma_{p}$ for the error of peak area calculation including $\varepsilon_{\text {Simpson }}$ from Eq. 10

$$
\sigma_{p}=\sqrt{\sigma_{A_{t o t}}^{2}+\sigma_{A_{b g}}^{2}+\epsilon_{\text {Simpson }}^{2}}=\sqrt{A_{\text {tot }}+A_{\text {bg }}+\epsilon_{\text {Simpson }}^{2}}
$$

with $A_{b g}$ the portion of the total peak area $A_{t o t}$ attributed to the background. With these considerations, it is now possible to automatically calculate the number of measured electrons, i.e., the area $A_{p} \pm \sigma_{p}$ for all found peak intervals.

\subsection{Quantitative Isotope Analysis}

Determination of precise and accurate abundances of isotopes is also of high interest in various basic and applied research fields, e.g. environmental science, analysis of plants or agricultural products, medical cell analyses, radioactive waste control, forensic analyses, geochemical analyses, including radio-isotope geochronology [10]. The investigations of isotope abundances in materials of solar system bodies are of particular importance to cosmochemistry and planetary science [33]. The quantitative measurements of the element and isotope abundances of matter from planetary objects provide critical constraints on events and their chronology in the early stages of the solar system formation and even later on.

Every element with more than one stable isotope has its own isotope abundance distribution. The isotope distribution can provide insight in different physical and chemical processes, as has been mentioned above. When carrying out quantitative isotope analyses, special attention has to be paid to possible isobaric interferences of isotopes of different elements.

The isotope abundances are simply obtained by calculating the ratio of the isotope's areas. To quantify the accuracy of the calculated isotope abundances, generally certified reference materials (CRM) are used for reference or in case of a terrestrial sample, natural (terrestrial) isotope abundance can be assumed. The relative accuracy of isotope ratio is then defined as

relative accuracy of isotope ratio $=\frac{\text { |experimental isotope ratio-reference isotope ratio| }}{\text { reference isotope ratio }}$

where the reference isotope ratio is either terrestrial [34] or from a certified material, e.g. from National Institute of Standards and Technology (NIST). 


\subsection{Automatic Analysis Software}

For a fully automatic data analysis software, the individual analysis steps that have been presented above, have to be linked together in a structured way. A flow-chart of the individual processes and presented algorithms is illustrated in Fig. 3.

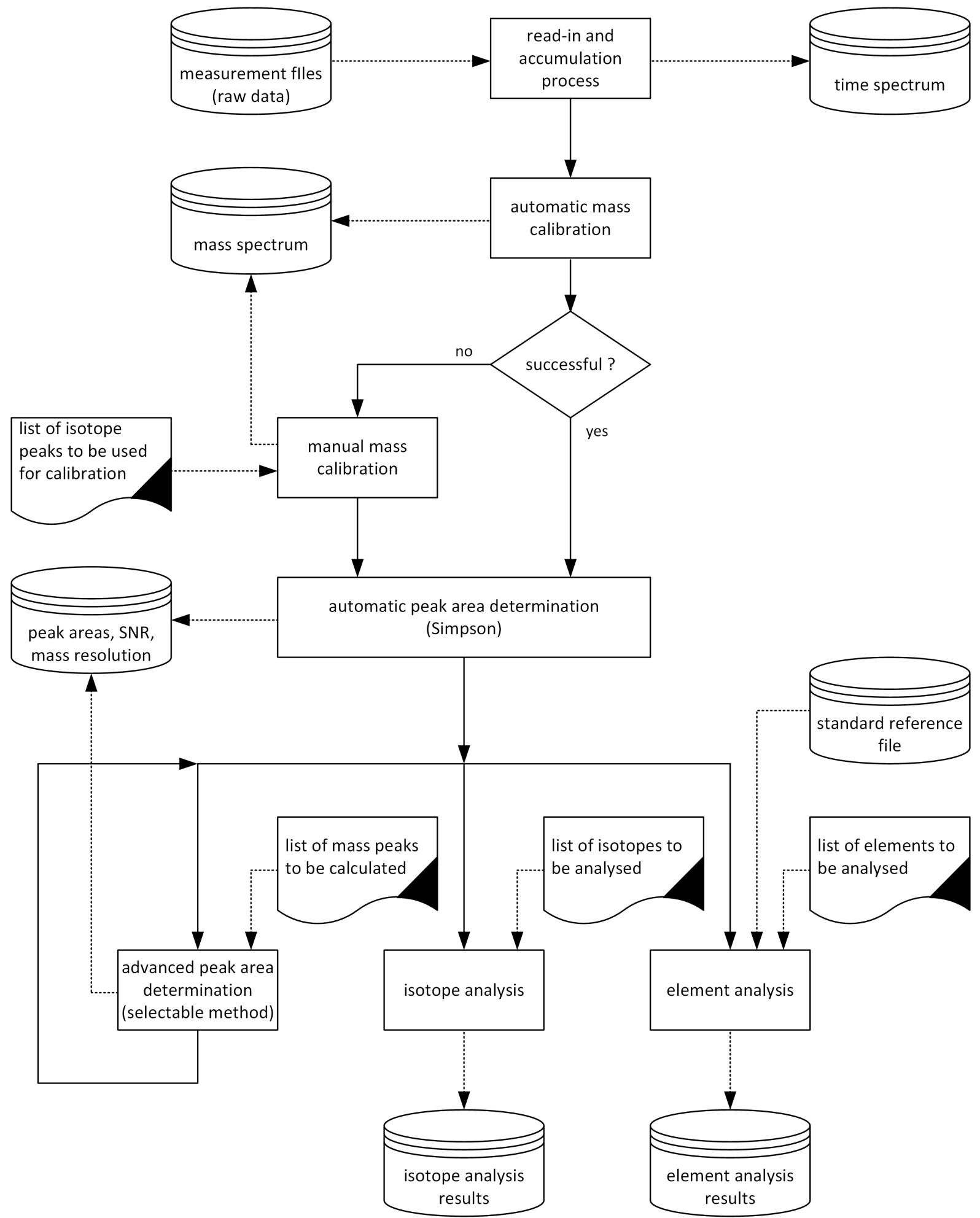

Figure 3: Flow-chart of the implemented functions for fully automated data analysis of a TOF measurement.

As described in Sec. 3.1, the read-in and accumulation process of the data are executed to generate the TOF spectrum from the measurement files. Subsequently, the automatic mass calibration of the TOF spectrum is initiated, providing the mass spectrum for further analysis. 
If the automatic mass calibration is not successful, a manual mass calibration is initiated. In this procedure, the software user is asked to provide a list of isotopes for calibration. Afterwards, automatic peak area determination by Simpson integration (see Sec. 3.2) of all mass peaks is executed, leading to the files containing all information that is needed for quantitative analysis. For keeping our software as universally applicable as possible, the possibility for applying other integration algorithms (MC integration, Gauss fit, EMG fit, Voigt fit and Lorentz fit) was kept. In the presented flow-chart, this is described as advanced peak area determination, where the user has to select the desired algorithm. Based on the results from the automatic peak area determination or from advanced peak area determination, either isotope analysis or element analysis can be carried out for a set of user defined elements and/or isotopes. This includes the computation of quantitative element abundances [35] or isotope ratios [19] and the according graphic accounts as an output.

With our software tool, quantitative data analysis of a measured data set can be done fully automated by the guidance of a trained user within minutes. In addition, the presented algorithms and processes can easily be adapted to every other TOF mass spectrometer (which has been done successfully in [36]), since all functions have been implemented in a modular way with universal interfaces and all used constants are editable on top level.

\section{Data Analysis Results and Discussion}

\subsection{Peak Area Calculation Method}

To compare the described numerical integration methods to other implemented fitting functions, the relative accuracy of the isotope ratios with respect to the terrestrial abundance (see Eq. 14 in Sec. 3.3) has been calculated for several isotopes of $\mathrm{Ne}$ and $\mathrm{Kr}$ with the NIM instrument. To cover a wide variety of mass peaks, the ${ }^{78} \mathrm{Kr}$ isotope with a low SNR and high mass, the ${ }^{80} \mathrm{Kr}$ isotope with a medium SNR and high mass, as well as the ${ }^{20} \mathrm{Ne}$ isotope with a high SNR and low mass and the ${ }^{22} \mathrm{Ne}$ isotope with a low SNR and low mass have been evaluated. Since ${ }^{21} \mathrm{Ne}$ is below the noise limit and the mass resolution in this measurement is too low for accurate automatic analysis of ${ }^{82} \mathrm{Kr},{ }^{83} \mathrm{Kr},{ }^{84} \mathrm{Kr}$ and ${ }^{86} \mathrm{Kr}$, terrestrial abundances have been assumed for these isotopes. The corresponding analysis of different fitting functions and numerical integration methods, using the above described peak window finding algorithm is illustrated in Fig. 4.

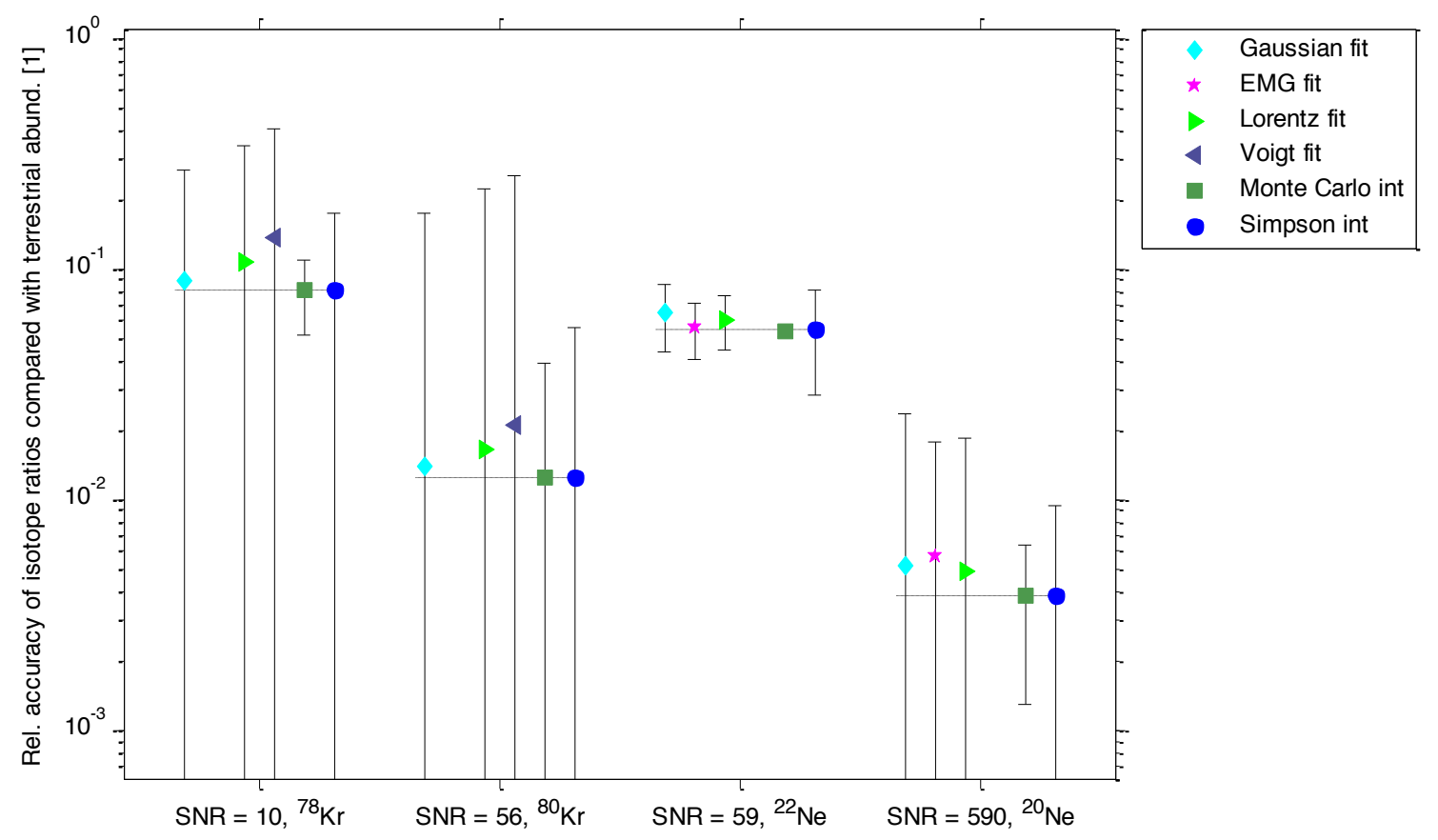


Figure 4: Accuracy of $\mathrm{Ne}$ and $\mathrm{Kr}$ isotopes relative to terrestrial abundances from [34], with respect to SNR. A missing data point indicates that the corresponding fitting method failed for this specific isotope.

It can be seen from Fig. 4 that the results with the highest accuracy are obtained with the numerical methods MC integration and Simpson integration. In each of the cases analysed, the deviation of the analysis result from the terrestrial abundance is smallest for these two methods. Both methods, MC integration and Simpson integration, approximately yield the same results. For the fitting models, we find that the Voigt function matches least to the measured mass peaks and the EMG function, as well as Voigt function failed to fit for some of the isotope peaks. Furthermore, the calculation time has to be considered, which is best for the Simpson integration with $<0.5 \mathrm{~s}$ for all listed isotopes. About $3 \mathrm{~s}$ are needed for the calculation by the algorithms using a fitting method. In contrast, the MC integration takes considerably more time, namely $8 \mathrm{~h}$ for all listed isotopes. From this investigation, it can be concluded that numerical Simpson integration is the most suitable method for our purpose of automatic peak area calculation. Not only is it very accurate, but it is also extremely robust and fast in comparison with all other discussed methods.

\subsection{Dependence of Accuracy of Isotope Ratios on SNR}

The analysis of the relative accuracy of isotope ratios as a function of the SNR shows an inverse proportional relationship between the relative accuracy of isotope ratios and SNR. This correlation is expected. Under the assumption of constant peak widths, the SNR is proportional to the peak areas. In Fig. 5 the relative isotope accuracy, assuming terrestrial abundances, is shown as a function of the SNR. Blue data points in Fig. 5 represent the measured data. In addition, the results from simulated spectra with Gaussian peaks, using SNR, mass resolution and a uniform distributed noise level equivalent to the measurement are illustrated by the red squares. The measurement results, as well as the simulation results, are in good agreement with the trend line, which indicates a proportionality $\mathrm{SNR}^{-1}$. Figure 5 demonstrates that relative accuracies for isotope ratios from worst case 1, for mass peaks right at the noise level $(S N R=1)$, down to $10 \mathrm{ppm}$ can be achieved for a dynamic range of $10^{5}$, i.e. $\mathrm{SNR}=10^{5}$. 


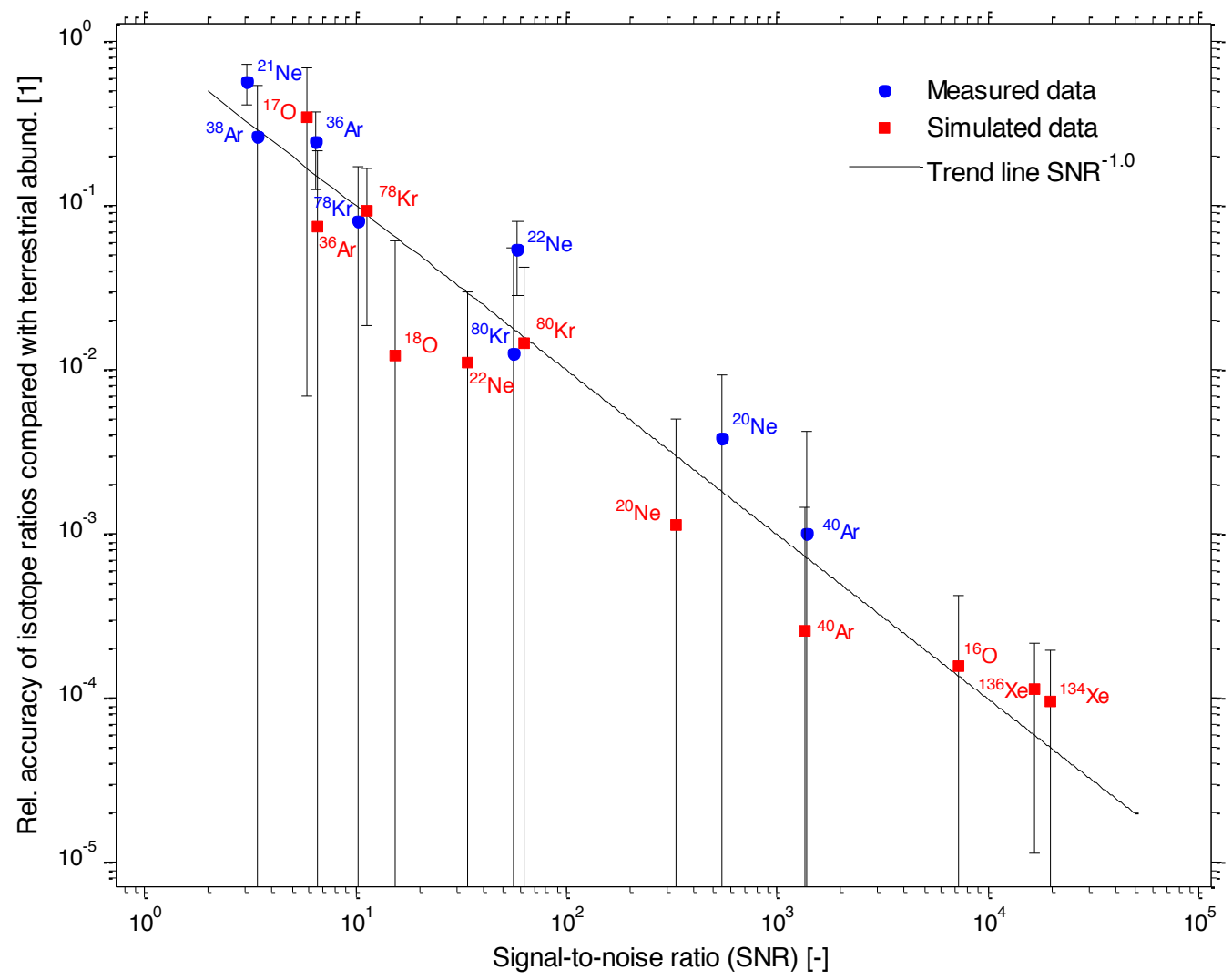

Figure 5: Relative accuracies of isotope ratios, derived by comparison to terrestrial abundance from [34], versus SNR for different isotopes of a noble gas measurement with the NIM instrument.

\subsection{Dependence of Accuracy of Isotope Ratios on Mass Resolution}

For a Gaussian peak, the SNR is proportional to the area of the peak and inversely proportional to the width of the peak, if we consider the maximum of the Gaussian peak at its mean value. Regarding the mass resolution $m / \Delta m, \Delta m$ can be represented by the width of the peak in the case of a Gaussian function. Therefore, for a constant peak area, i.e., a constant number of detected ions, the relative accuracy of isotope ratios also is inversely proportional to the mass resolution. This correlation is shown in Fig. 6 . The graph shows the analysis of relative accuracies of the isotope ratios ${ }^{17} \mathrm{O},{ }^{22} \mathrm{Ne},{ }^{84} \mathrm{Kr}$ and ${ }^{124} \mathrm{Xe}$ obtained from simulated mass spectra with Gaussian mass peaks, using SNR and a uniform distributed noise level equivalent to the measurement for different mass resolutions. From Fig. 6 it can be seen that the proportionality only holds above the minimum mass resolution that is needed by the analysis software to resolve two adjacent mass peaks. As described in Eq. 6 in Sec. 3.2, this minimum mass resolution is proportional to the mass itself; higher masses therefore need also higher mass resolution. 


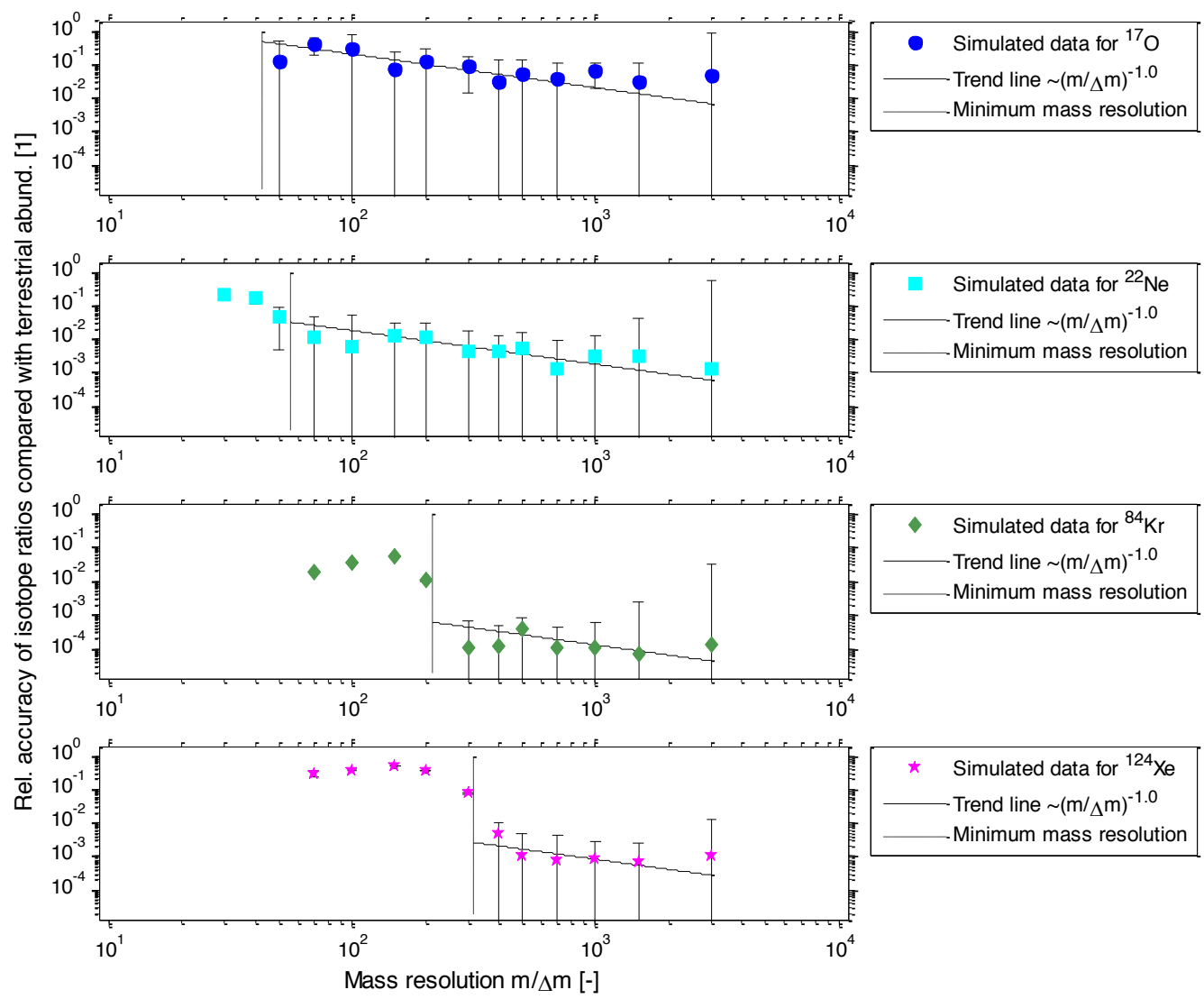

Figure 6: Relative accuracy of isotope ratios ${ }^{17} \mathrm{O},{ }^{22} \mathrm{Ne},{ }^{84} \mathrm{Kr}$ and ${ }^{124} \mathrm{Xe}$ calculated for different mass resolutions. The results were obtained from simulated spectra with Gaussian peaks.

\subsection{Dependence of Accuracy of Isotope Ratios on Sample Width}

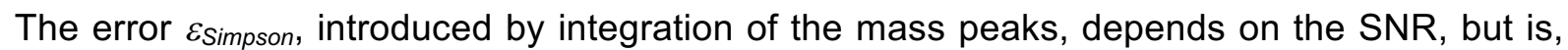
in addition, related to the sample width $T_{s}$, (see Eq. 10). This leads to the presumption that the accuracy of the peak calculation depends on $T_{s}$ as well. For testing this correlation, the noble gas measurements have been resampled several times and analysed with respect to the accuracy of the $\mathrm{Ne}$ and $\mathrm{Kr}$ isotopes. The original measurement data were acquired with $T_{s}=0.25 \mathrm{~ns}$. In addition, also simulated spectra with Gaussian peaks, using SNR, mass resolution and a uniform distributed noise level equivalent to the measurement have been analysed. The results are presented in Fig. 7. From the graph, the correlation of the relative accuracy of isotope ratios and the sample width is obvious. In all cases the correlation of $T_{s}^{0.5}$, marked by a corresponding trend line, fits both, the measured and resampled data, as well as the simulated spectra. 

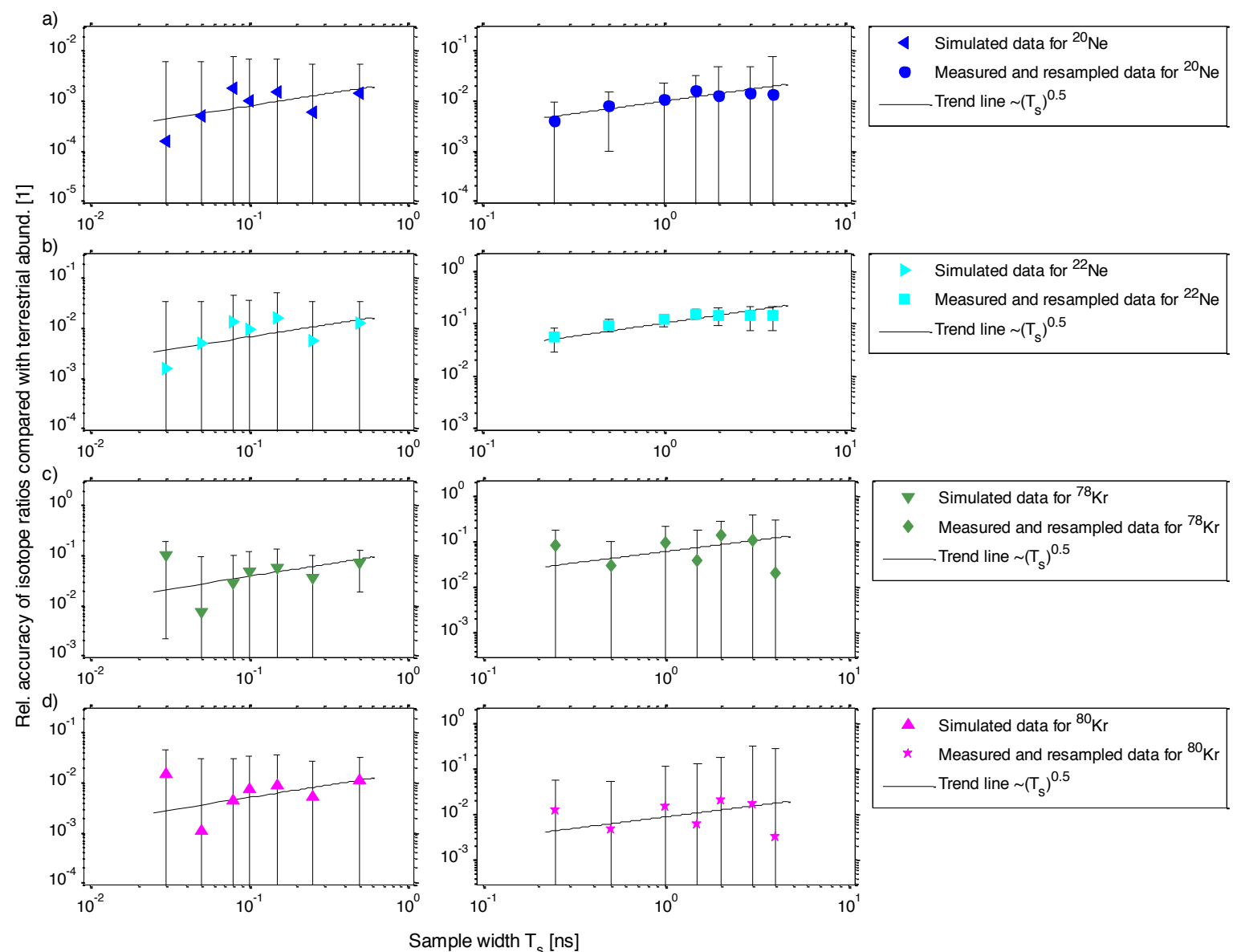

Figure 7: Relative accuracy of isotope ratios calculated for different sample widths, for ${ }^{20} \mathrm{Ne}$ (a), ${ }^{22} \mathrm{Ne}(\mathrm{b}),{ }^{78} \mathrm{Kr}$ (c) and ${ }^{80} \mathrm{Kr}$ (d). The panels on the left hand side show results of simulated spectra with Gaussian peaks using SNR, mass resolution and a uniform distributed noise level equivalent to the measurement.

A possible explanation for this trend can be given by considering oversampling and quantisation noise. It is known, e.g. [37], that for each bit of additional resolution, the signal has to be oversampled by a factor of four. Thus, the sampling frequency $f_{s}$, can be written as

$$
f_{s}=4^{n_{s}} \cdot f_{N y q},
$$

with $f_{N y q}$ representing the Nyquist frequency and $n_{s}$ the additional number of bits due to oversampling. Assuming a minimum quantisation noise of one digit, the maximum possible signal-to-quantisation noise ratio $S Q N R_{\max }$ covers the full range of resolution with $N$ the total number of bits

$$
S Q N R_{\text {max }}=2^{N}=2^{n_{0}+n_{s}},
$$

where the total number of bits is a sum of the effective ADC bits $n_{0}$ and the additional number of bits due to oversampling $n_{s}$. Finally, by combining Eq. 15 and Eq. 16, the sampling frequencies can be expressed by the corresponding Nyquist sample width $T_{N y q}$ and the sample width $T_{s}$ leading to

$$
S Q N R_{\text {max }}=2^{n_{0}} \cdot\left(\frac{f_{S}}{f_{N y q}}\right)^{\frac{1}{2}}=2^{n_{0}} \cdot\left(\frac{T_{S}}{T_{N y q}}\right)^{-\frac{1}{2}},
$$

which explains the correlation observed (Fig. 7). 


\section{Summary and Conclusions}

A fully automated mass spectrum analysis software has been developed for measurement data obtained with a TOF mass spectrometer. With the help of the automatic mass calibration algorithm, data input, reduction and analysis, to the point of peak area determination of all detected mass peaks, can be performed in a robust and reliable way. Additionally, implemented routines for isotope and element analyses allow a trained user to derive quantitative results from the measurement data within minutes. This automatic spectrum analysis software enables the analysis of hundreds of measurements in short time, but also yields very accurate results as well as extremely high reproducibility. In addition, the presented algorithms and processes can easily be adapted to every other TOF mass spectrometer system, since all functions have been implemented in a modular way with universal interfaces and all used constants editable on top level.

By applying a numerical Simpson integration and developing the corresponding error estimation, we were able to carry out accurate isotope abundance analysis with up to $10 \mathrm{ppm}$ relative accuracy. We showed that Simpson integration yields more accurate results compared to common curve fitting models. Additionally, the Simpson integration method is more suitable concerning the robustness of the algorithm compared to the common curve fitting models. Furthermore, Simpson integration is of advantage due to short computation times. It allows the calculation of peak area for a whole spectrum in a few seconds, whereas the equally accurate and robust MC integration needs several hours to carry out the same analysis. Therefore, the Simpson integration discussed in this article is currently considered to be the most suitable method for automatic peak area determination.

A peak window finding algorithm, a prerequisite for accurate isotope abundance calculation, was developed. For this algorithm, we found a robust local minimum and maximum searching algorithm being most suitable. The algorithm is capable to successfully cover a range from SNR $>1$ up to maximum SNR, which is about $10^{5}$ for the NIM instrument used for this study presented here. Together with the according routines for background correction, SNR and mass resolution calculation, the automatic peak area calculation algorithm based on Simpson integration has been implemented in the analysis software. The automated and robust peak area calculation algorithm not only allows very accurate data analysis, but also guarantees unbiased data processing and highest reproducibility of the results.

Based on the results obtained with simulated mass spectra, we conclude that the quantitative isotope analysis using automatic peak area detection yields results with a relative accuracy up to 1 per mille for the most abundant isotopes and up to $100 \mathrm{ppm}$ for a SNR of $10^{4}$. We showed that the relative accuracy of isotope ratios is in fact proportional to $S N R^{-1}$, since the calculated peak area is linearly coupled with SNR. This turns out to be useful for the estimation of relative accuracy of isotope ratios at a certain abundance level, for example to predict an estimation error for radio-isotope geochronology of material, as presented in [19]. However, the minimum mass resolution is essential for obtaining highest accuracies. Moreover, we observed that relative accuracy of isotopes also is inversely proportional to mass resolution, as SNR is. Mass resolution is linked to the SNR, since better mass resolution results in smaller, but higher peaks and thus larger SNR, assuming constant peak area, i.e., constant number of detected ions.

Additionally, we found that the relative accuracy of isotope ratios is proportional to the sampling width $T_{\mathrm{s}}^{-0.5}$. This has been shown for measured and resampled data, as well as for simulated data. Concluding from this result, better accuracies can be achieved by higher sampling frequency of the used ADC system. Nevertheless, dynamic range should be given priority by choosing an appropriate ADC, i.e., data acquisition system, since relative accuracy stronger depends on SNR than on sampling width. 


\section{References}

[1] J. Vago, O. Witasse, H. Svedhem, P. Baglioni, A. Haldemann, G. Gianfiglio, T. Blancquaert, D. McCoy, and R. de Groot, "ESA ExoMars Program: The Next Step in Exploring Mars," ISSN 0038-0946, Solar System Research, 2015, Vol. 49, No. 7, pp. 518-528.

[2] O. Grasset, M.K. Dougherty, A. Coustenis, E.J. Bunce, C. Erd, D. Titov, M. Blanc, A. Coates, P. Drossart, L.N. Fletcher, H. Hussmann, R. Jaumann, N. Krupp, J.-P. Lebreton, O. Prieto-Ballesteros, P. Tortora, F. Tosi, T. Van Hoolst, "JUpiter ICy moons Explorer (JUICE): An ESA mission to orbit Ganymede and to characterise the Jupiter system," Planetary and Space Science, 78 (2013), 1-21.

[3] J.P. Grotzinger, J. Crisp, A.R. Vasavada, R.C. Anderson, C.J. Baker, R. Barry, D.F. Blake, P. Conrad, K.S. Edgett, B. Ferdowski, R. Gellert, J.B. Gilbert, M. Golombek, J. Gómez-Elvira, D.M. Hassler, L. Jandura, M. Litvak, P. Mahaffy, J. Maki, M. Meyer, M.C. Malin, I. Mitrofanov, J.J. Simmonds, D. Vaniman, R.V. Welch, R.C. Wiens, "Mars Science Laboratory Mission and Science Investigation," Space Sci Rev (2012) 170, 556.

[4] P. Wurz, D. Abplanalp, M. Tulej, M. lakovleva, V.A. Fernandes, A. Chumikov, and G. Managadze, "Mass Spectrometric Analysis in Planetary Science: Investigation of the Surface and the Atmosphere," Sol. Sys. Res. 46 (2012) 408-422.

[5] W. B. Brinckerhoff, G. G. Managadze, R. W. McEntire, A. F. Cheng, W. J. Green, "Laser time-of-flight mass spectrometry for space, Review of Scientific Instruments," American Institute of Physics, 2000.

[6] G.G. Managadze, I.Y. Shutyaev, "Laser Ionisation Mass Analysis," Chemical Analysis, Vol. 124 Wiley, New York, 1993.

[7] U. Rohner, J. A. Whitby and P. Wurz, "A miniature laser ablation time-of-flight mass spectrometer for in situ planetary exploration," Meas. Sci. Technol. 14 (2003) 21592164.

[8] U. Rohner, J. A. Whitby, P. Wurz and S. Barabash, "Highly miniaturized laser ablation time-of-flight mass spectrometer for a planetary rover," Review of Scientific Instruments, Vol. 75, Nr. 5, 2004.

[9] V. Grimaudo, P. Moreno-Garcia, A. Riedo, S. Meyer, M. Tulej, M.B. Neuland, M. Mohos, C. Gütz, S.R. Waldvogel, P. Wurz and P. Broekmann, "Toward ThreeDimensional Chemical Imaging of Ternary $\mathrm{Cu}-\mathrm{Sn}-\mathrm{Pb}$ Alloys Using Femtosecond Laser Ablation/lonization Mass Spectrometry", Anal. Chem., 89, 2017, 1632 - 1641.

[10] J. S. Becker, "Inorganic Mass Spectrometry: Principles and Applications," John Wiley \& Sons Ltd., 2007.

[11] S. Scherer, K. Altwegg, H. Balsiger, J. Fischer, A. Jäckel, A. Korth, M. Mildner, D. Piazza, H. Rèmeand and P. Wurz, "A novel principle for an ion mirror design in time-offlight mass spectrometry, " Int. J. Mass Spectr. 251, 73-81, 2006.

[12] H. Balsiger, K. Altwegg, P. Bochsler, P. Eberhardt, J. Fischer, S. Graf, A. Jäckel, E. Kopp, U. Langer, M. Mildner, J. Müller, T. Riesen, M. Rubin, S. Scherer, P. Wurz, S. Wüthrich, E. Arijs, S. Delanoye, J. De Keyser, E. Neefs, D. Nevejans, H. Rème, C. Aoustin, C. Mazelle, J.-L. Médale, J.A. Sauvaud, J.-J. Berthelier, J.-L. Bertaux, L. Duvet, J.-M. Illiano, S.A. Fuselier, A.G. Ghielmetti, T. Magoncelli, E.G. Shelley, A. Korth, K. Heerlein, H. Lauche, S. Livi, A. Loose, U. Mall, B. Wilken, F. Gliem, B. Fiethe, T.I. Gombosi, B. Block, G.R. Carignan, L.A. Fisk, J.H. Waite, D.T. Young, and H. Wollnik, "ROSINA - Rosetta Orbiter Spectrometer for Ion and Neutral Analysis," Space Science Review 128 (2007), 745-801.

[13] L. Hofer, P. Wurz, A. Buch, M. Cabane, P. Coll, D. Coscia, M. Gerasimov, D. Lasi, A. Sapgir, C. Szopa, M. Tulej, "Prototype of the gas chromatograph-mass spectrometer to investigate volatile species in the lunar soil for the Luna-Resurs mission," Planetary and Space Science 111 (2015) 126-133.

[14] S. Meyer, M. Tulej, P. Wurz, "Mass spectrometry of planetary exospheres at high relative velocity: direct comparison of open- and closed-source measurements," Geosci. Instrum. Method. Data Syst., 6, 1-8, 2017. 
[15] M.B. Neuland, S. Meyer, K. Mezger, A. Riedo, M. Tulej, P. Wurz, "Probing the Allende meteorite with a miniature Laser-Ablation Mass Analyser for space application," Planetary and Space Science 101 (2014) 196-209.

[16] A. Riedo, V. Grimaudo, P. Moreno-Garcia, M.B. Neuland, M. Tulej, P. Broekmann and P. Wurz, "Laser Ablation/lonisation Mass Spectrometry: Sensitive and quantitative chemical depth profiling of solid materials", CHIMIA, 70, 2016, $268-273$.

[17] M. Tulej, M. lakovleva, I. Leya, P. Wurz, "A miniature mass analyser for in-situ elemental analysis of planetary material - performance studies," Anal. Bioanal. Chem. (2011) 399, 2185-2200.

[18] A. Riedo, A. Bieler, M. Neuland, M. Tulej, P. Wurz, "Performance evaluation of a miniature laser ablation time-of-flight mass spectrometer designed for in-situ investigations in planetary space research," Jou. Mass Spectr. 48 (2013) 1-15.

[19] A. Riedo, S. Meyer, B. Heredia, M. Neuland, A. Bieler, M. Tulej, I. Leya, M. lakovleva, K. Mezger, P.Wurz, "Highly accurate isotope composition measurements by a miniature laser ablation mass spectrometer designed for in situ investigations on planetary surfaces," Planetary and Space Science 87 (2013) 1-13.

[20] A. Riedo, M.B. Neuland, S. Meyer, M. Tulej, P. Wurz, "Coupling of LMS with a fs-laser ablation ion source: elemental and isotope composition measurements," J. Anal. At. Spectrom., 28, 1256, 2013.

[21] M. Tulej, A. Riedo, M.B. Neuland, S. Meyer, D. Lasi, D. Piazza, N. Thomas, and P. Wurz, "A miniature instrument suite for in situ investigation of the composition and morphology of extraterrestrial materials," Geostand. Geoanal. Res, 38 (2014) 441-466.

[22] M. Tulej, A. Neubeck, M. Ivarsson, A. Riedo, M.B. Neuland, S. Meyer, P. Wurz, "Chemical Composition of Micrometer-Sized Filaments in an Aragonite Host by a Miniature Laser Ablation/lonization Mass Spectrometer," Astrobiology, Volume 15, Number 8, 2015.

[23] D. Abplanalp, P. Wurz, L. Huber, I. Leya, E. Kopp, U. Rohner, M. Wieser, L. Kalla, and S. Barabash, "A neutral gas mass spectrometer to measure the chemical composition of the stratosphere," Adv. Space Res., 44, 870-878, 2009.

[24] D. Abplanalp, P. Wurz, L. Huber, and I. Leya, "An Optimised Compact Electron Impact Ion Source for a time-of-flight mass spectrometer," Int. Jou. Mass Spectrom., 294 (2010), 33-39.

[25] U. Rohner, "Development of Highly Miniaturised Laser-Mass-Spectrometers for the in situ Analysis of Planetary Surfaces," PhD Thesis, University of Berne, 2004.

[26] M.B. Neuland, "In situ mass spectrometry for planetary exploration: Quantitative chemical composition measurements on planetary surfaces," PhD Thesis, University of Berne, 2016.

[27] P. Du, W.A. Kibbe, S.M. Lin, "Improved Peak Detection in Mass Spectrum by Incorporating Continuous Wavelet Transform-based Pattern Matching," Bioinformatics Advance Access published July 4, 2006.

[28] G. Wells, H. Prest, C.W. Russ, "Technical Note from Agilent Technologies: Signal, Noise and Detection Limits in Mass Spectrometry," 5990-7651EN, May 3, 2011.

[29] P.R. Bevington, D.K. Robinson, "Data Reduction and Error Analysis," Third Edition, McGraw-Hill Companies, New York, 2003.

[30] W.H. Press, S.A. Teukolsky, W.T. Vetterling, B.P. Flannery, "NUMERICAL RECIPES, The Art of Scientific Computing," Third Edition, Cambridge University Press, New York, 2007.

[31] B. Fornberg, "Generation of Finite Difference Formulas on Arbitrarily Spaced Grids," Mathematics of Computation (1988) 51 184, 699-706, 1988.

[32] H. Stöcker, "Taschenbuch mathematischer Formeln und moderner Verfahren," Harri Deutsch Verlag, 4. korrigierte Auflage, 1999.

[33] I. N. Tolstikhin, J. D. Kramers, "The evolution of matter, Cambridge University Press, New York, 2008.

[34] J. Meija, T. B. Coplen, M. Berglund, W. A. Brand, P. De Bièvre, M. Gröning, N. E. Holden, J. Irrgeher, R. D. Loss, Th. Walczyk and Th. Prohaska, "Isotopic compositions 
of the elements 2013 (IUPAC Technical Report)," Pure Appl. Chem. 2016; 88(3): 293306.

[35] Neuland, M.B., Grimaudo, V., Mezger, K., Moreno-Garcia, P., Riedo, A., Tulej., M. and Wurz, P., "Quantitative measurement of the chemical composition of geological standards with a miniature laser ablation/ionization mass spectrometer designed for insitu application in space research," MMMeas. Sci. Technol. 27 (2016), 035904, 13pp.

[36] D.M. Paardekooper, G. Fedoseev, A. Riedo and H. Linnartz, "A novel approach to measure photodesorption rates of interstellar ice analogues. The photodesorption rate of CO ice reinvestigated," Astron. Astrophys., 596, 2016, A72, 1 - 7.

[37] SILICON LABS, "Improving ADC Resolution by Oversampling and Averaging," Silicon Laboratories Inc., 400 West Cesar Chavez, Austin, TX 78701, USA, AN118, Rev. 1.3 7/13, 2013. 